\title{
Awareness of World Health Organization methicillin- resistant Staphylococcus aureus guidelines at Alexandria University hospitals
}

\author{
G.S. Soliman, ${ }^{7}$ R.M. Abu-Youssef, ${ }^{1}$ B.F. Saleib, ${ }^{7}$ A.M. El-Moughazi' and A. Zaki²
}

$$
\text { جيرمين سالوح سالدلائل الإرشادية لمنظمة الصحة العانية راندة محمود أبو يوسف، بيشوي فهم العنقوديات الذهبية المقاومة للميثيسيلين في مستشفيات جامعة الإسكند محمد المغازي، عادل زكية }
$$

الخلاصـة: تهدف دراستنا المستعرضة هذه إلى تقييم معارف العاملين الطبيين في مستشفيات جامعة الإسكندرية حول طرق السراية، والمعرفة بالدلائل

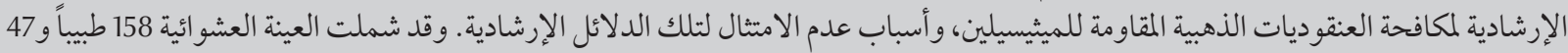

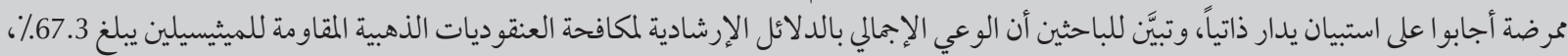

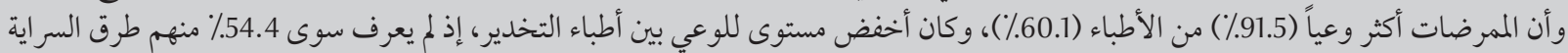

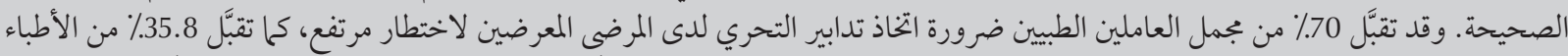

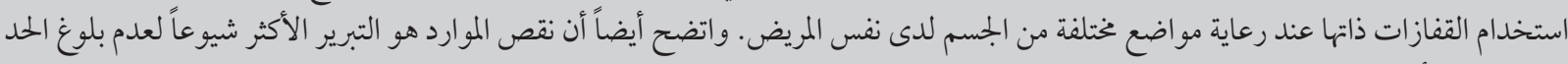

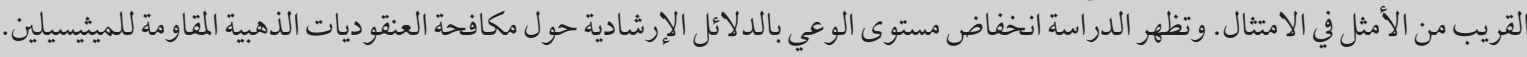

ABSTRACT This cross-sectional study aimed to assess knowledge of routes of transmission, awareness of MRSA control guidelines and reasons for non-adherence to guidelines among medical staff at Alexandria University hospitals. A random sample of 158 physicians and 47 nurses answered a self-administered questionnaire. Overall awareness of MRSA control guidelines was $67.3 \%$, and nurses were significantly more aware than physicians (91.5\% versus $60.1 \%$ ). The lowest awareness level was among anaesthesiologists; only 54.4\% knew the correct transmission routes. Among medical staff overall, 70.0\% accepted the necessity of screening measures for highrisk patients and $35.8 \%$ of doctors accepted the use of the same pair of gloves when caring for different body sites on an individual patient. Lack of resources was the most common justification for suboptimum adherence. The study showed low awareness levels of MRSA-related guidelines.

Sensibilisation aux recommandations de l'Organisation mondiale de la Santé sur la lutte contre Staphylococcus aureus résistant à la méthicilline dans des hôpitaux universitaires d'Alexandrie

RÉSUMÉ La présente étude transversale visait à évaluer la connaissance des voies de transmission, la sensibilisation aux recommandations sur la lutte contre Staphylococcus aureus résistant à la méthicilline et les motifs du non respect de ces recommandations par le personnel médical des hôpitaux universitaires d'Alexandrie. Un échantillon randomisé de 158 médecins et 47 infirmières ont répondu à un auto-questionnaire. Globalement, la sensibilisation aux recommandations sur la lutte contre Staphylococcus aureus résistant à la méthicilline était de $67,3 \%$, et le personnel infirmier était beaucoup plus sensibilisé que les médecins (91,5\% contre 60,1\%). Le niveau de sensibilisation le plus faible a été retrouvé chez les anesthésistes. Seuls 54,4\% de ces derniers connaissaient les voies de transmission correctes. Au sein du personnel médical globalement, 70,0\% avaient accepté la nécessité de mesures de dépistage pour les patients à haut risque mais 35,8 \% des médecins reconnaissaient utiliser une seule paire de gants même lors de soins prodigués sur différents sites corporels d'un même patient. Le manque de ressources était la justification la plus fréquente pour le respect insuffisant de ces recommandations. L'étude a mis en évidence les faibles niveaux de sensibilisation aux recommandations sur la lutte contre Staphylococcus aureus résistant à la méthicilline. 


\section{Introduction}

Methicillin-resistant Staphylococcus aureus (MRSA) is now a major healthcareacquired pathogen, accounting for up to $40 \%$ of staphylococcal infections in developed countries $[1,2]$. Colonization occurs by contact with contaminated wounds, dressings and inanimate objects of infected patients, with another individual's colonized intact skin and by inhalation of aerosolized droplets from chronic nasal carriers [3-5]. Risk factors include surgical site infection, antibiotic use, prolonged hospitalization, intensive care, haemodialysis and proximity to others with MRSA colonization or infection [6-8].

The worldwide MRSA prevalence varies considerably, from less than $1 \%$ in Scandinavia to up to $40 \%$ in Japan, Israel and elsewhere in Europe [7,9]. In a 2007 study on invasive isolates from southern and eastern Mediterranean countries, the highest proportions of MRSA isolates were reported by Jordan, Egypt and Cyprus. Egypt reported a rate of $52 \%$ in comparison with other Mediterranean countries such as Lebanon, Morocco, Algeria, Tunisia and Malta, which reported rates of $12 \%, 19 \%$, $45 \%, 18 \%$ and $50 \%$ respectively, while Jordan and Cyprus reported 56\% and $55 \%$ respectively [10]. A 2009 study in Alexandria Main University hospitals reported that of 100 S. aureus isolates, 71 were MRSA, mostly from patients in the intensive care unit (ICU) [11].

Countries that have failed to implement early MRSA surveillance and control measures have struggled to control MRSA epidemics $[12,13]$. The level of awareness of MRSA infection control varies in different countries. A study of medical staff in the United Kingdom (UK) found that knowledge about numerous aspects of MRSA and its management was deficient [14]. In Switzerland a study of adherence to the guideline recommendation of hand washing [15] reported only a $48 \%$ adherence rate of health care workers [16].
To the best of our knowledge, there has not been any published study in Egypt investigating the level of awareness among health care workers on MRSA control guideline recommendations. Taking this into account, our study was developed with to assess the level of awareness of health care staff, both physicians and nurses, at Alexandria University hospitals concerning MRSA guideline recommendations, and to determine the reasons, if any, for nonadherence.

\section{Methods}

\section{Study setting and sample}

The period of data collection of this cross-sectional study was from September to November 2010 at both the Main University hospital and the Paediatrics and Obstetrics hospitals of University of Alexandria, serving the public sector of the city of Alexandria, Egypt. The units where MRSA was expected to be an important issue were selected, namely: the general ICU, the paediatric ICU (PICU) and neonatal ICU (NICU), the pulmonology medicine department, the anaesthesiology department, the cardiothoracic surgery department and the coronary care unit (CCU). The units totalled 200 beds and the total number of medical staff working in these units was 192 physicians and 75 registered nurses (graduates of the faculty of nursing). Nurses who had graduated from the school of nursing, whose education was in Arabic, did not meet the eligibility requirements for a survey conducted in English. Department heads were informed of the study prior to data collection. A random sample of physicians and registered nurses working in the different selected units were asked to participate in the study.

Those who agreed to complete the questionnaire included a total of 158 physicians and 47 registered nurses. A pilot study of 25 medical staff personnel was conducted to ensure comprehension and clarity of the questions prior to questionnaire administration.

\section{Data collection}

Each participant was asked to complete the pre-tested, self-administered questionnaire, which included demographic data such as age, sex, year of graduation, and the unit where he/she worked. The investigator was present to answer any questions.

Initially, using multiple choice questions the questionnaire inquired about knowledge about routes of transmission and the most important risk factor for MRSA infection. Concerning each individual's awareness about the presence of MRSA guidelines, participants were asked: "Have you heard of any guidelines on MRSA and can you mention any?" Medical staff aware of the existence of at least one guideline and able to provide at least one source was deemed "aware"; otherwise "not aware". Those who were not aware of these guidelines did not complete the second part of the questionnaire regarding guideline recommendations.

Regarding their detailed knowledge of the guideline recommendations, each of the World Health Organization (WHO), recommendations was transformed into a true/false question, and the responses were classified as "correct" or "incorrect" [17].

In the final part of the survey, medical staff was asked in an open question format to mention their justification(s) for non-adherence (if any) to MRSA control recommendations in their units. The questionnaire ended by enquiring about methods by which they kept themselves updated on guidelines.

\section{Statistical analysis}

The data were analysed using SPSS, version 15.0. Frequency distributions presenting percentages of different groups of health care professionals interviewed regarding MRSA control guidelines were tabulated. Differences 
between percentages of physicians and nurses who gave correct answers for questions covering awareness of guidelines and guideline recommendations were tested using the chi-squared test. For all statistical analyses, the level of significance was set at $P<0.05$.

\section{Results}

Table 1 shows the distribution of respondents (158 physicians and 47 registered nurses) across the different departments. The mean age of the 205 respondents was 32 (SD 7) years; 99 (48.3\%) were males and 109 (51.7\%) females

\section{Knowledge about MRSA routes of transmission}

Table 2 shows the respondents' awareness of MRSA routes of transmission and main risk factor for MRSA infection in the different units. There was no significant difference between nurses and physicians in their awareness of MRSA routes of transmission (57.4\% vs $54.5 \%$ respectively, $P>0.05)$ but significantly more nurses were aware of the main risk factor $(23.4 \%$ vs $20.3 \%$ respectively, $P<0.05$ ). This was particularly evident in the general ICU, CCU and pulmonology departments. In the general ICU $65.2 \%$ nurses versus $61.2 \%$ of physicians correctly identified MRSA transmission routes, namely airborne mechanisms, contaminated surfaces and skin contact. In the CCU, $60.0 \%$ of nurses were aware of the correct MRSA transmission routes, while $55.6 \%$ of physicians identified them. Likewise, $40.0 \%$ of pulmonology nurses knew how MRSA is transmitted, while only $29.2 \%$ of pulmonology physicians knew these routes.

\section{Awareness of MRSA guidelines}

Medical staff in the NICU and PICU overall showed an $85.7 \%$ awareness rate of the MRSA guidelines. This was the highest rate reported from the departments. The general ICU also demonstrated a relatively high rate of awareness $(73.3 \%)$. In contrast, the rate of guideline awareness was only $45.0 \%$ in the anaesthesiology department ( $\mathrm{Ta}$ ble 3). In each unit, the rate of awareness of nurses was higher than that of physicians, and overall $91.5 \%$ of nurses and $60.1 \%$ of physicians were aware of the guidelines $(P>0.001)$.

One section of the questionnaire was dedicated specifically to the level of awareness on MRSA guideline recommendations. Notably, $90.7 \%$ of nurses and $60.0 \%$ of physicians were aware of the importance of screening measures to detect early cases and implement appropriate precautions $(P<0.001)$. Similarly, $100 \%$ of nurses and $90.5 \%$ of physicians knew that the detection of cases is mandatory, especially if there is referral from another hospital $(P<$ 0.05). Concerning treatment issues, $86.0 \%$ of the nurses acknowledged that airborne precautions should be implemented when caring for a patient with MRSA pneumonia, while $68.4 \%$ of physicians responded similarly $(P<$ 0.05) (Table 4).

More nurses (83.7\%) than physicians (64.2\%) acknowledged that using only one pair of gloves for each patient when caring for more than one body site is inadequate procedure $(P<0.05)$. However, $9.3 \%$ of nurses and $16.8 \%$ of physicians accepted the protocol that the utilization of gloves renders handwashing optional after the care of the patient, according to the severity of the patient's condition $(P>0.05)$ (Table 4).

The 3 most common justifications given by medical staff for suboptimum adherence to MRSA guideline implementation were: lack of resources (58.2\%), lack of staff awareness (32.9\%) and non-compliance/negligence (20.3\%).

Clinicians used a myriad of sources for learning about and continuing to be updated on MRSA guidelines, namely the Internet in general (23.8\%), UpToDate (20.7\%), peers (16.1\%), books, journals, Medscape, pharmaceutical pamphlets and tutors.

\section{Discussion}

To the best of our knowledge, this is the first study in Egypt to assess the awareness level of MRSA guidelines, common routes of transmission and the most important guideline recommendations

\begin{tabular}{lcccc}
\hline Table 1 Distribution of the study respondents across different hospital departments & & & \multicolumn{1}{c}{ Total } \\
Hospital unit & $\begin{array}{c}\text { Physicians } \\
(\boldsymbol{n}=\mathbf{1 5 8})\end{array}$ & $\begin{array}{c}\text { Nurses } \\
(\boldsymbol{n}=\mathbf{4 7})\end{array}$ & No. 205) \\
& No. & n/a & 20 & 9.8 \\
Anaesthesiology & 20 & 2 & 15 & 7.3 \\
Cardiothoracic surgery & 13 & 5 & 23 & 11.2 \\
Coronary care unit & 18 & 23 & 90 & 43.9 \\
General intensive care unit & 67 & 5 & 29 & 14.1 \\
Pulmonology medicine & 24 & 12 & 28 & 13.7 \\
Neonatal and paediatric intensive care units & 16 & & No. \\
\hline
\end{tabular}

$n / a=$ not applicable. 


\begin{tabular}{|c|c|c|c|c|}
\hline \multirow[t]{2}{*}{ Unit } & \multicolumn{2}{|c|}{$\begin{array}{l}\text { Correct MRSA } \\
\text { transmission route }^{\mathrm{a}}\end{array}$} & \multicolumn{2}{|c|}{$\begin{array}{l}\text { Most important risk } \\
\text { factor for MRSA } \\
\text { infection }^{\mathrm{b}}\end{array}$} \\
\hline & No. & $\%$ correct & No. & $\%$ correct \\
\hline General intensive care unit & 56 & 62.2 & 18 & 20.0 \\
\hline Coronary care unit & 13 & 56.5 & 4 & 17.4 \\
\hline $\begin{array}{l}\text { Neonatal and paediatric intensive } \\
\text { care units }\end{array}$ & 18 & 64.3 & 8 & 28.6 \\
\hline Anasthesiology & 7 & 35.0 & 3 & 15.0 \\
\hline Cordiothoracic surgery & 10 & 66.7 & 6 & 40.0 \\
\hline Internal medicine chest & 9 & 31.0 & 4 & 13.8 \\
\hline$P$-value & \multicolumn{2}{|c|}{$<0.05$} & \multicolumn{2}{|c|}{$>0.05$} \\
\hline
\end{tabular}

${ }^{a}$ Correct answer: airborne droplets, contaminated surfaces, and skin contact; ${ }^{b}$ Correct answer: open skin wounds.

among health care workers at Alexandria University hospitals. Although the study included only hospital departments in which MRSA was considered a major potential infectious threat, the general awareness level towards MRSA guidelines was unexpectedly low (67.3\%). Only $20.3 \%$ of physicians and $23.4 \%$ of nurses correctly identified the most important risk factor for infection, i.e. open skin wounds. Similarly, the correct main routes of transmission were identified by slightly more than half of the medical staff (55.0\%). The contrast between the relatively high rate of awareness of MRSA guidelines and the low rates of correct answers about risk factors and routes of transmission may suggest that most of the medical staff may hear about the existence of MRSA guidelines without actually having adequate, continual access to these guidelines. A Swiss study on hand-washing compliance revealed the consequences of this, in which a lack of awareness of the concepts of a procedure can lead to failure to perform it correctly [15]. Our findings are comparable with a UK study, where only $11.5 \%$ of medical staff correctly identified common risk factors for MRSA infection or colonization. Moreover, $25.9 \%$ of their staff knew the 2 most common sites of MRSA infection, namely blood and wound/ skin [14].

Our study showed discrepancies in awareness across different hospital departments as well as between physicians and nurses. Contrary to popular belief, nurses (91.5\%) were significantly more aware than physicians (60.1\%) about the presence of MRSA guidelines. The lowest awareness levels among physicians were found in the CCU and anaesthesiology units (33.3\% and $45.0 \%$ respectively), while the highest were in the NICU and PICU (75.0\%). Among the nurses, the lowest awareness was in the ICU $(82.6 \%)$, while $100 \%$ in the remaining departments were aware. As to MRSA recommendations, nurses had a significantly higher rate of correct answers compared with physicians about the necessity of screening measures as a strategy for MRSA control (90.7\% and $60.0 \%$ respectively). Surprisingly, $100 \%$ of nurses believed in the principle of early detection of cases, especially those referred from other health care facilities, while only $90.5 \%$ of physicians shared the same belief. Since isolation is a pillar of multi-drug resistant infection containment, an absolute consensus for this protocol was expected from medical staff, yet the study revealed that $8.4 \%$ of physicians and $4.7 \%$ of nurses did not acknowledge this protocol. Reasons behind the higher rates of infection knowledge and awareness of MRSA management practices among nurses compared with physicians should be investigated. Differences in the infection control syllabi at the faculty of medicine and faculty of nursing should

Table 3 Awareness of the guidelines on methicillin-resistant Staphylococcus aureus (MRSA) control: comparison of physicians and nurses in different hospital units

\begin{tabular}{|c|c|c|c|c|c|c|c|}
\hline \multirow[t]{2}{*}{ Hospital unit } & \multicolumn{2}{|c|}{ Total } & \multicolumn{2}{|c|}{ Physicians } & \multicolumn{2}{|c|}{ Nurses } & \multirow[t]{2}{*}{$P$-value } \\
\hline & No. in unit & $\%$ aware & No. in unit & $\%$ aware & No. in unit & $\%$ aware & \\
\hline $\begin{array}{l}\text { General intensive care } \\
\text { unit }\end{array}$ & 90 & 73.3 & 67 & 70.1 & 23 & 82.6 & $>0.05$ \\
\hline Coronary care unit & 23 & 47.8 & 18 & 33.3 & 5 & 100.0 & $<0.01$ \\
\hline $\begin{array}{l}\text { Neonatal and paediatric } \\
\text { intensive care units }\end{array}$ & 28 & 85.7 & 16 & 75.0 & 12 & 100.0 & $>0.05$ \\
\hline Anaesthesiology & 20 & 45.0 & 20 & 45.0 & $\mathrm{n} / \mathrm{a}$ & $\mathrm{n} / \mathrm{a}$ & \\
\hline Cardiothoracic surgery & 15 & 66.6 & 13 & 61.5 & 2 & 100.0 & $>0.05$ \\
\hline Pulmonology & 29 & 62.1 & 24 & 54.2 & 5 & 100.0 & $>0.05$ \\
\hline Total & 205 & 67.3 & 158 & 60.1 & 47 & 91.5 & $<0.001$ \\
\hline
\end{tabular}

$n / a=$ not applicable. 


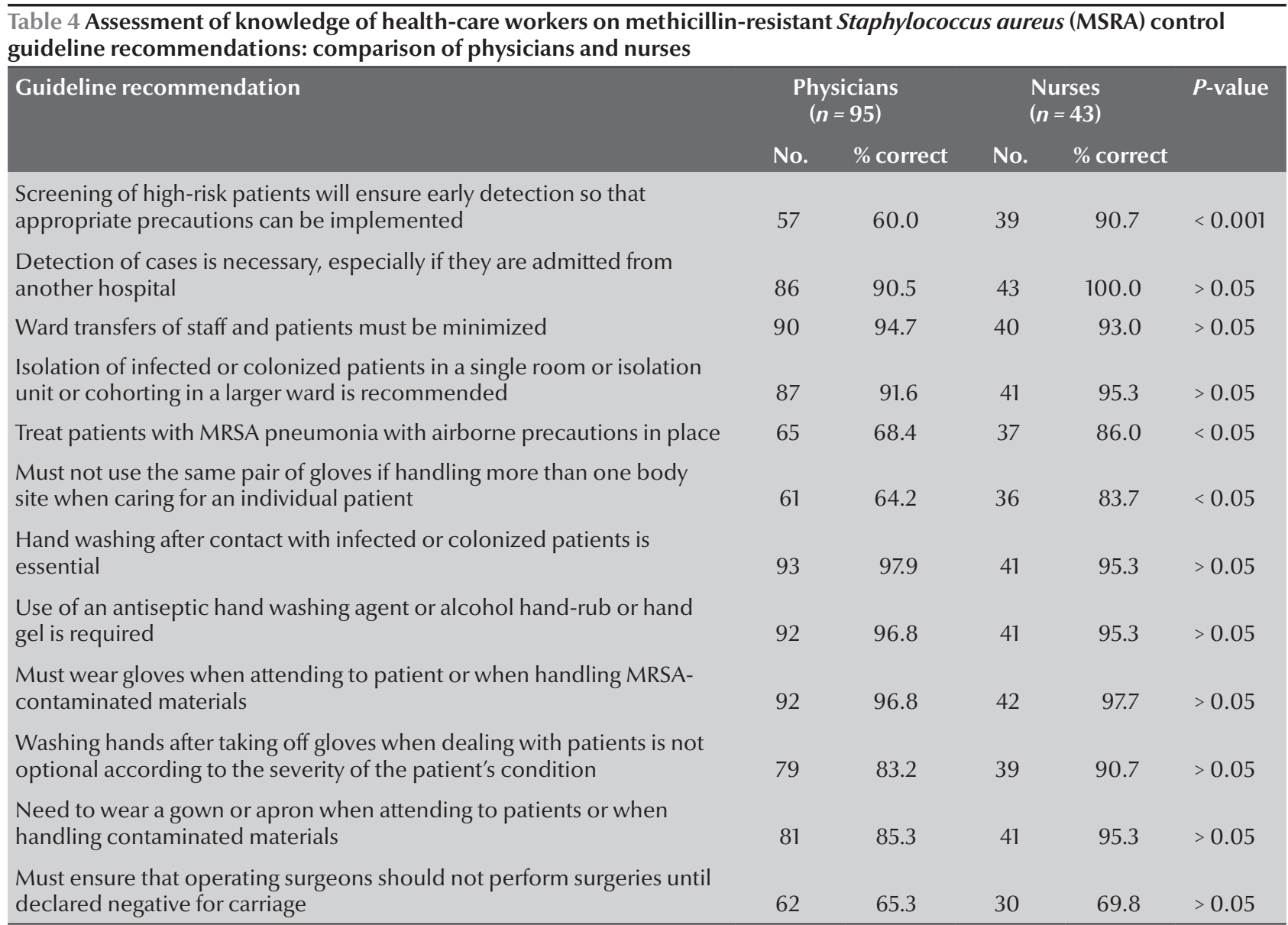

be revised as one of the possible reasons. According to the UK study, doctors were more competent in knowing treatment measures for MRSA patients, while nurses excelled in infection control practices [14]. A similar finding of the superior competence in infection control of nurses over doctors was in the Swiss paper on hand washing compliance in a teaching hospital. In 2834 observed opportunities for hand washing, there was a $48 \%$ compliance rate, of which the non-compliance was higher among doctors than nurses [15].

In addition to substantial variation of responses between physicians and nurses, our findings showed that individual departments differed considerably on many guideline recommendations, such as the necessity of airborne precautions when caring for a patient with MRSA pneumonia. The pulmonology department valued this protocol more than other departments.
Hence, protocols tailored according to the nature of work and the priorities of the various departments should be designed to ensure efficient protocol implementation. This is backed by a 2005 Dutch study on MRSA protocols in which a questionnaire and practical tests found that health care workers' knowledge of and attitudes towards protocols were adequate, yet they were inefficient at applying the protocols in their work circumstances, suggesting that it is impractical to use one MRSA protocol for all hospital staff due to the different decisions staff have to take according to their respective circumstances [18].

Our study showed that $35.8 \%$ of doctors and $16.3 \%$ of nurses considered it acceptable to use the same pair of gloves when caring for different body sites on each individual patient $(P>$ $0.05)$. These low percentages are comparable to the Swiss study, where hand washing compliance was found to be higher for low-risk procedures e.g. after wound care, and lower for procedures with a high risk of transmission e.g. $11 \%$ for care between dirty and clean body sites and $18 \%$ for before respiratory care [15].

Health care institutions striving for standard infection prophylaxis and management practice among their specialist as well as non-specialist staff members must invest their resources in improving generic knowledge of staff, as well as familiarizing them with the utilization of local guidance [14]. About one-third of our medical staff mentioned that lack of awareness was a cause for non-implementation of guidelines. This emphasizes the need for workshops and seminars to focus on key topic areas as well as issues assumed to be generic and trivial and which may therefore be overlooked when designing an infection control 
programme. The UK study showed that $68 \%$ of medical staff thought that more information was needed within their respective departments on the issues of MRSA colonization, infection, virulence, risk factors, general management and drug treatment, confirming that assumptions should not be made about adequate knowledge and expertise of staff in relation to MRSA [14]. Cabana et al's paper examining reasons why physicians do not follow clinical practice guidelines stated that the first step in improving clinicians' adherence to clinical practical guidelines is the attainment of thoroughly competent knowledge and attitudes, which culminates in the most sustainable change in clinician behaviour among all infection control strategies [19]. There are 4 critical sequential steps of any health education programme for ameliorating the behaviour of health care workers towards adhering to MRSA control guidelines: to be aware of the guidelines; to intellectually agree with them; to decide to adopt them in provided care; and to regularly and properly adhere to these guidelines, comprising the awarenessto-adherence model of clinical guideline compliance [20].

Despite the fact that MRSA guideline implementation by means of personal protective equipment and hand-washing stations is not costly, the most frequent justification given by medical staff for non-implementation in their departments was the lack of resources (58.2\%). This issue needs to be discussed with university hospital administrators.

In the UK study, only $2.3 \%$ of interviewees referred solely to the hospital's recommended resource for infection management consultation (a pocketsized prescribing guide booklet handed to all clinicians) [14]. This agrees with our study, where clinicians reported using a myriad of sources for learning about and staying updated on MRSA guidelines, such as (in decreasing order of frequency) the Internet in general, UpToDate, peers, books, journals, Medscape, pharmaceutical pamphlets and tutors. Hospital administrators and medical and nursing faculty staff need to develop a multi-specialty educational source by taking advantage of the varied sources to target key educational needs, incorporating them into undergraduate and postgraduate teaching systems and integrating them into the clinical decision-making of practitioners.

Some medical staff (17.7\%) stressed that work overload was a cause for decreased compliance with clinical guidelines. The Swiss study found that hand washing compliance decreases with increased patient care intensity [15]. This is supported by another study claiming that understaffing of wards decreases compliance with isolation measures [21]. Hospital administrators must be approached in order to address the demands of health care workers for work relief.

We do not known whether our results can be generalized to other regions or even other health care institutions in Egypt, since the infrastructure and work organization of individual health care institutions influence the knowledge, outlook and attitude of health care workers.

\section{Conclusions}

The study showed relatively low levels of awareness of MRSA guidelines among health care workers in Alexandria University hospitals. Concerted efforts by the hospital management administration should be exerted to maintain an influential infection control team whose responsibility is the reinforcement of guideline implementation as well as the undertaking of periodic health education programmes for both the specialist and non-specialist staff members of health care institutions. Medical professionals must be aware of the potential danger of MRSA infection, since countries that did not implement early MRSA surveillance and control measures have struggled to control MRSA epidemics.

Competing interests: None declared.

\section{References}

1. Healthcare-associated infections (HAI). Centers for Disease Control and Prevention [online] (http://www.cdc.gov/ ncidod/dhqp/ar_mrsa_ca_public.html, accessed 4 April 2013).

2. Graham PL 3rd, Lin SX, Larson EL. A U.S. population-based survey of Staphylococcus aureus colonization. Annals of Internal Medicine, 2006, 144:318-325.

3. Barber M. Methicillin-resistant staphylococci. Journal ofClinical Pathology, 1961, 14:385-393.

4. Coello $\mathrm{R}$ et al. Prospective study of infection, colonization and carriage of methicillin-resistant Staphylococcus aureus in an outbreak affecting 990 patients. European Journal of Clinical Microbiology and Infectious Diseases, 1994, 13:74-81.

5. Miller LG et al. Clinical and epidemiologic characteristics cannot distinguish community-associated methicillin-resistant Staphylococcus aureus infection from methicillin-suscep- tible $S$. aureus infection: a prospective investigation. Clinical Infectious Diseases, 2007, 44:471-482.

6. Davis KA et al. Methicillin-resistant Staphylococcus aureus (MRSA) nares colonization at hospital admission and its effect on subsequent MRSA infection. Clinical Infectious Diseases, 2004, 39:776-782.

7. Voss A et al. Methicillin-resistant Staphylococcus aureus in Europe. European Journal of Clinical Microbiology and Infectious Diseases, 1994, 13:50-55.

8. Thompson RL, Cabezudo I, Wenzel RP. Epidemiology of nosocomial infections caused by methicillin-resistant Staphylococcus aureus. Annals of Internal Medicine, 1982, 97:309-317.

9. Sader HS et al. Antimicrobial susceptibility of gram-positive bacteria isolated from European medical centres: results of the Daptomycin Surveillance Programme (2002-2004). Clinical Microbiology and Infection, 2006, 12:844-852. 
10. Borg MA et al. Prevalence of methicillin-resistant Staphylococcus aureus (MRSA) in invasive isolates from southern and eastern Mediterranean countries. Journal of Antimicrobial Chemotherapy, 2007, 60:1310-1315.

11. Sadaka SM et al. Evaluation of different methods for the rapid diagnosis of methicillin-resistance in Staphylococcus aureus. African Journal of Microbiology Research, 2009, 3:49-55.

12. Simor AE et al. The evolution of methicillin-resistant Staphylococcus aureus in Canadian hospitals: 5 years of national surveillance. Canadian Medical Association Journal, 2001, 165:21-26.

13. Gastmeier P et al. [Surveillance of nosocomial infections in intensive care units. Current data and interpretations.] Surveilt lance nosokomialer Infektionen in Intensivstationen: Aktuelle Daten und Interpretationen. Wiener Klinische Wochenschrift, 2003, 115(3-4):99103.

14. Easton PM et al. Infection control and management of MRSA: assessing the knowledge of staff in an acute hospital setting. Journal of Hospital Infection, 2007, 66:29-33.

15. Pittet D, Mourouga P, Perneger TV. Compliance with hand, washing in a teaching hospital. Annals of Internal Medicine, 1999, 130:126-130.
16. Larson EL. APIC guideline for handwashing and hand antisepsis in health care settings. American Journal of Infection Control, 1995, 23:251-269.

17. Practical guidelines for infection control in health care facilities. Geneva, World Health Organization, 2004.

18. Van Gemert-Pijnen J et al. Performance of methicillin-resistant Staphylococcus aureus protocols in Dutch hospitals. American Journal of Infection Control, 2005, 33:377-384.

19. Cabana MD et al. Why don't physicians follow clinical practice guidelines? A framework for improvement. Journal of the American Medical Association, 1999, 282:1458-1465.

20. Pathman DE et al. The awareness-to-adherence model of the steps to clinical guideline compliance. The case of pediatric vaccine recommendations. Medical Care, 1996, 34:873-889.

21. Pettinger A, Nettleman MD. Epidemiology of isolation precautions. Infection Control and Hospital Epidemiology, 1991, 12:303-307.

\section{The evolving threat of antimicrobial resistance - Options for action}

In 2012, WHO launched the book The evolving threat of antimicrobial resistance - Options for action. This publication examines the experiences with interventions which address the growing threat of antimicrobial resistance (AMR), describes the lessons learnt along the way and highlights the gaps still remaining. It draws attention to areas where knowledge is lacking and where urgent action is still needed.

The aim of the book is to raise awareness about AMR and to stimulate further efforts to meet the recommendations outlined in the WHO 2001 Strategy for Containment of AMR and in the 2011 World Health Day policy package. It does so by examining the current situation, and setting out what has been done and what could still be done around the world, in high-, middle- and low-income countries. While much of what is summarized in this publication is well known to the scientific community, yet awareness at the political level is essential, but often lacking. A specific objective is therefore to encourage policy-makers and the global community to commit to intensified action against AMR.

Further information about this and other WHO publications is available at: http://apps.who.int/bookorders/anglais/ home1.jpp 